\title{
ANÁLISIS DE LOS GRUPOS DE INVESTIGACIÓN COLOMBIANOS EN CIENCIAS ECONÓMICAS DESDE UNA PERSPECTIVA DE GÉNERO*
}

\author{
JENNY PAOLA LIS GUTIÉRREZ** \\ UNIVERSIDAD CENTRAL (COLOMBIA)
}

Recibido/ Received/ Recebido: 21/05/2012 - Aceptado/ Accepted / Aprovado: 01/10/2012

\begin{abstract}
Resumen
A partir del cálculo de indicadores de género (distribución horizontal, distribución vertical, índice de feminidad, índice de masculinidad, índice de Duncan, índice de segregación e índice de contribución al sexismo) y el uso de representaciones cartográficas temáticas, se presenta una aproximación cuantitativa sobre la brecha de género y la segregación departamental en el liderazgo de los grupos de investigación colombianos en las áreas de Economía y Administración, de acuerdo con la información disponible hasta junio de 2012. Se halló que el liderazgo femenino de grupos de investigación en las dos disciplinas es inferior al promedio nacional; las tasas de graduación de mujeres de pregrado y posgrado entre 2000 y 2010 no se reflejan en la composición de los grupos de investigación; la mayor parte de grupos de investigación liderados por mujeres se encuentran en categoría D; en la mayoría de los departamentos hay líderes de grupo de ambos sexos, pero superan en cantidad los hombres a las mujeres; al igual que en el contexto nacional los grupos de investigación, para ambos casos, se concentran en Bogotá, Antioquia y Valle del Cauca.
\end{abstract}

Palabras clave: Género, Paridad, Indicadores de género, Estudios sociales de la ciencia.

\section{ANALYSIS OF COLOMBIAN RESEARCH GROUPS IN ECONOMIC SCIENCES FROM A GENDER PERSPECTIVE}

\begin{abstract}
From the calculation of gender indicators (horizontal distribution, vertical distribution, feminine index, masculinity index, Duncan index, segregation index and contribution to sexism index) and the use of thematic cartographic representations, a quantitative approximation is presented on gender branch and departmental segregation in the leadership of Colombianresearchin economic and management areas, according to the available information until June 2012. The findings showed that feminine leadership of research groups in the two disciplines of study is inferior to national average; the graduation rates of undergraduate and graduate studies between 2000 and 2010 are not reflected in the composition of research groups; the majority of research groups with feminine leaders are in category D; most of the department have both male and female leaders, but male over exceeds women; and this is similar at national level for both cases, the groups are focused in Bogotá, Antioquia and Valle del Cauca.
\end{abstract}

Keywords: Gender, Parity, Gender indicators, Social studies of science.

\footnotetext{
Artículo de investigación, producto del proyecto "Fortalecimiento de capacidades relacionadas con la equidad de género en la educación superior" (NUFFIC - NICHE/COL 061/091) financiado por la Netherlands organisation for international cooperation in higher education (NUFFIC) y la Netherlands Initiative for Capacity development in Higher Education (NICHE). El artículo es un producto conexo del trabajo realizado en el marco del proyecto en el Instituto de Estudios Sociales Contemporáneos y en la Facultad de Ciencias Administrativas, Económicas y Contables de la Universidad Central.

**: Magíster en Análisis de problemas políticos, económicos e internacionales (Universidad Externado de Colombia), Magíster en Sociedades contemporáneas comparadas Europa-América Latina (Paris III- Sorbonne Nouvelle), especialidad en Geografía y Planeación (IHEAL), Economista (Universidad Nacional de Colombia). Se desempeña actualmente como docente de la Universidad Central y miembro del Grupo de Estudios Económicos de la Superintendencia de Industria y Comercio. Correo electrónico: jlisg@ucentral.edu.co
} 


\title{
ANÁLISE DOS GRUPOS DE PESQUISA COLOMBIANOS EM CIÊNCIAS ECONÔMICAS DESDE UMA PERSPECTIVA DE GÊNERO
}

\section{Resumo}

\begin{abstract}
A partir do cálculo de indicadores de gênero (distribuição horizontal, distribuição vertical, índice de feminilidade, índice de masculinidade, índice de Duncan, índice de segregação e índice de contribuição ao sexismo) e o uso de representações cartográficas temáticas, apresenta-se uma aproximação quantitativa sobre a brecha de gênero e a segregação estadual na liderança dos grupos de pesquisa colombianos nas áreas de Economia e Administração, de acordo com a informação disponível até junho de 2012. Encontrou-se que a liderança feminina de grupos de pesquisa nas duas disciplinas é inferior à média nacional; as taxas de conclusão de curso de mulheres em graduações e pós-graduações entre 2000 e 2010 não se refletem na composição dos grupos de pesquisa; a maior parte de grupos de pesquisa liderados por mulheres se encontram em categoria $\mathrm{D}$; na maioria dos estados há líderes de grupo de ambos os sexos, mas os homens superam as mulheres em quantidade; da mesma forma como no contexto nacional, os grupos de pesquisa, para ambos os casos, concentram-se em Bogotá, Antioquia e Valle del Cauca.
\end{abstract}

Palavras chave: Gênero, Paridade, Indicadores de gênero, Estudos sociais da ciência.

Liz, J. (2012) Análisis de los grupos de investigación colombianos en ciencias económicas desde una perspectiva de género. En: Revista de la Facultad de Ciencias Económicas de la Universidad Militar Nueva Granada. rev.fac.cienc.econ, XX (2).

JEL: O30, O54, D63, I23, I24.

\section{Introducción}

La perspectiva de género puede ser entendida como "la metodología y los mecanismos que permiten identificar, cuestionar y valorar las desigualdades, la exclusión y la discriminación por razones de sexo" (Buquet Corleto, Cooper, Rodríguez \& Botello, 2006, 309). En Colombia, los estudios sociales de la ciencia que incluyen el enfoque de género son pocos (Daza, 2010; Olaya, 2003; González \& Pérez, 2002; Tovar, 2002; Arango, 2006) y no hay ninguno aplicado específicamente a la investigación en Economía.

A partir de estadísticas básicas, indicadores de género (distribución horizontal, distribución vertical, índice de feminidad, índice de masculinidad, índice de Duncan, índice de segregación e índice de contribución al sexismo) y representaciones mediante cartografía temática, se pretende identificar si existen tanto brechas de género como segregación geográfica, en el liderazgo de los grupos de investigación colombianos en economía y administración.

Para ello, el trabajo está organizado en cuatro partes. En la primera sección se presenta una revisión conceptual sobre estudios de género aplicados a indicadores de ciencia y tecnología en Colombia. Posteriormente, se explica la metodología empleada y los indicadores que se calculan en este trabajo. En el tercer segmento, se presentan los resultados (nacionales y aplicados específicamente al caso de los grupos de investigación en economía y administración). Finalmente, se formulan las conclusiones.

\section{Revisión conceptual}

Aunque la relación entre género y ciencia ha sido interés de algunos investigadores colombianos, la mayor parte de trabajos se concentran en el análisis agregado y no en áreas específicas. Por ejemplo, el trabajo de Daza-Caicedo (2010) presenta un análisis descriptivo sobre estadísticas e indicadores sobre la presencia de hombres y mujeres en el SNCTI durante el período 2000-2010, mostrando con cifras las brechas de género que aún existen. Con esta misma aproximación se elaboraron los trabajos de Tovar (2002, 2004, 2005), en los cuales se presentaban y discutían de manera crítica los indicadores de género aplicados a estadísticas nacionales de ciencia y tecnología. 
Daza-Caicedo \& Pérez-Bustos (2008) reflexionaron sobre los indicadores de género, en el campo de la ciencia y la tecnología en Colombia, a la luz de estudios latinoamericanos, proponiendo 4 estrategias para el caso colombiano: i) reformular las preguntas y objetivos de los indicadores de género tradicionales; ii) elaboración de indicadores de redes sociales y otros relacionados con lo que ocurre al interior de los grupos sociales; iii) elaboración de encuestas de percepción pública de la ciencia y análisis cuantitativos de consumo y difusión de la ciencia; iv) incluir la perspectiva de género en las políticas públicas.

Por su parte, González \& Pérez (2002) elaboraron una aproximación epistemológica sobre la relación género/ciencia. Por su parte, el trabajo de Olaya (2003) analizó los resultados de la convocatoria de grupos y centros de investigación a la luz de la participación de hombres y mujeres. Igualmente, Munévar, Arana \& Agudelo (2006), realizan un análisis sobre la productividad académica en la Universidad Nacional y el desempeño de las docentes mujeres en la institución.

Los análisis sobre brecha salarial entre hombre y mujeres en Colombia, son un poco más frecuentes. El más recientemente es el de Cepeda \& Barón (2012), quienes a partir de microdatos de la Base de Graduados del Observatorio Laboral y empleando la metodología Oaxaca-Blinder para descomponer la brecha salarial promedio, encontraron una relación entre el área de estudios de los recién graduados y las brechas salariales por género. En Colombia, en promedio "el núcleo de estudio explica salarios entre 3 y $4 \%$ más altos para los hombres" (Cepeda \& Barón, 2012, 13). Otros trabajos en esta misma dirección son el de Gutiérrez (2010) y Cano \& Orozco (2011).

A nivel internacional sobresalen los estudios de Papadópulos \& Radakovich (2006) y Lemarchand (2010). Este último, a partir de cifras de la UNESCO (2009), analiza las estadísticas de participación de mujeres en el número total de investigadores del planeta. Lemarchand encuentra que a nivel mundial, para 2009, esta proporción era 29\%, pero en América Latina era de $46 \%$.

Igualmente, son múltiples los documentos que analizan la relación entre docencia y género. Por ejemplo, los trabajos de Zapata Galindo (2010) sobre equidad de género y personal científico en universidades alemanas; Bustos \& Blázquez (2003), Buquet Corleto et al. (2006), Buquet Corleto, Cooper \& Rodríguez (2010) Buquet Corleto, Hernández \& Jiménez (2011), para el caso de la UNAM y sus diferentes dependencias; García Guevara (1992) sobre las características socio-demográficas de las mujeres académicas y análisis de género en la Universidad de Guadalajara (García Guevara, 2004, 2009; Palomar, 2004), Escolano (2006, 2009), para el rol de las académicas en caso de las universidades españolas; Bailyn (2003) sobre las mujeres académicas en el MIT, entre otros.

\section{Metodología empleada}

En este trabajo se presentan tanto estadísticas básicas como indicadores de género, aplicados al análisis de la variable sexo del líder de los grupos de investigación en Economía y Administración visibles en la plataforma ScienTi de Colciencias a junio de 2012. Igualmente, se analiza la composición de los grupos de investigación en Economía clasificados en categoría $\mathrm{A} 1$ y $\mathrm{A}$, de acuerdo con la convocatoria de 2010.

Las estadísticas básicas corresponden a la desagregación de los datos por sexo, con el propósito de realizar análisis comparativos básicos (descriptivo y relacional). En el segundo caso de los indicadores de género, se trata de "variables de análisis que describen la situación de las mujeres y hombres en la sociedad. Su utilización supone una aproximación a la situación o presencia de mujeres y hombres, así como la incidencia de determinados factores que implican diferencias de comportamientos entre unas $y$ otros" (Monge, 2006, 52).

La base fuente de información utilizada fueron los registros sobre grupos de investigación colombianos, disponibles en la plataforma ScienTi ${ }^{1}$.

1 Dado que la información de Scienti no puede exportarse, fue necesario copiar registro por registro para obtener la base de datos completa de los grupos de investigación. 
Tabla 1. Indicadores de género calculados para los grupos de investigación²

\begin{tabular}{|c|c|c|c|}
\hline Indicador & Explicación & Cálculo & Interpretación \\
\hline $\begin{array}{l}\text { Distribución } \\
\text { horizontal }\end{array}$ & $\begin{array}{l}\text { Participación relativa de } \\
\text { mujeres y hombres en un } \\
\text { ambiente i determinado. }\end{array}$ & $\begin{array}{l}\text { Dh }=\text { XijTi *100 } \\
\text { Donde xij es la cantidad de personas en el am- } \\
\text { biente i del sexo j; y Ti es el total de personas en } \\
\text { el ambiente i. }\end{array}$ & $\begin{array}{l}\text { Porcentaje de personas del sexo j dentro del total de la } \\
\text { población en el ambiente i. }\end{array}$ \\
\hline $\begin{array}{l}\text { Distribución } \\
\text { vertical }\end{array}$ & $\begin{array}{l}\text { Concentración de los indi- } \\
\text { viduos de un mismo sexo } \\
\text { entre los distintos nom- } \\
\text { bramientos, actividades, } \\
\text { carreras o planteles. }\end{array}$ & $\begin{array}{l}\text { Dv }=[(\mathrm{Xij} / \mathrm{Tj})]^{\star} 100 \\
\text { Siendo xij la cantidad de personas en el nombra- } \\
\text { miento, ocupación, carrera o plantel i del sexo j; } \\
\text { y Tj es el total de personas del sexo j. }\end{array}$ & $\begin{array}{l}\text { Porcentaje de personas del sexo j en el nombramiento, } \\
\text { actividad, carrera o plantel i, dentro del total de la población } \\
\text { del sexo j. }\end{array}$ \\
\hline $\begin{array}{l}\text { Índice de } \\
\text { feminidad }\end{array}$ & $\begin{array}{l}\text { Número de mujeres por } \\
\text { cada } 100 \text { hombres dentro } \\
\text { de una población o con- } \\
\text { junto en estudio. }\end{array}$ & $\begin{array}{l}\text { Es el cociente entre el número de mujeres y el } \\
\text { número de hombres en una población } \\
\text { IF }=X m X h \text { *100 } \\
\text { Donde xm es la cantidad de mujeres y xh es la } \\
\text { cantidad de hombres dentro de una población } 0 \\
\text { conjunto en estudio }\end{array}$ & Número de mujeres por cada 100 hombres. \\
\hline $\begin{array}{l}\text { Índice de } \\
\text { masculinidad }\end{array}$ & $\begin{array}{l}\text { Número de hombres por } \\
\text { cada } 100 \text { mujeres dentro de } \\
\text { una población o conjunto } \\
\text { en estudio. }\end{array}$ & $\begin{array}{l}\text { Es el cociente entre el número de hombres y el } \\
\text { número de mujeres en una población } \\
\text { IF }=X h X m \text { *100 } \\
\text { Donde xm es la cantidad de mujeres y xh es la } \\
\text { cantidad de hombres dentro de una población } 0 \\
\text { conjunto en estudio. }\end{array}$ & Número de hombres por cada 100 mujeres. \\
\hline $\begin{array}{l}\text { Índice de Duncan } \\
\text { (indicador de } \\
\text { segregación) }\end{array}$ & $\begin{array}{l}\text { Porcentaje de mujeres y de } \\
\text { hombres que tendrían que } \\
\text { cambiar de nombramien- } \\
\text { to, de puesto o de carrera } \\
\text { para que hombres y mujeres } \\
\text { estuvieran distribuidos de } \\
\text { manera equitativa. }\end{array}$ & $\begin{array}{l}\text { ID = 12Mujeres en la ocupación iEmpleo total } \\
\text { femenino-Hombres en la ocupación iempleo } \\
\text { total masculino }\end{array}$ & $\begin{array}{l}\text { Con este índice se calcula la diferencia entre la proporción de } \\
\text { individuos del grupo minoritario }(X) \text { y la proporción del resto de } \\
\text { población. Este índice puede tomar cualquier valor comprendido } \\
\text { entre } 0 \text { y } 1 \text {, y cuanto más alto sea el valor, expresa mayor grado } \\
\text { de segregación. Donde no hay segregación, el índice de Duncan } \\
\text { es igual a cero. El valor } 1 \text { implica segregación completa; cada } \\
\text { ocupación es completamente masculina o femenina. }\end{array}$ \\
\hline $\begin{array}{l}\text { Índice de } \\
\text { disimilaridad de } \\
\text { Duncan (IDD) por } \\
\text { sexo }\end{array}$ & $\begin{array}{l}\text { Proporción de hombres } \\
\text { (mujeres) que deberían } \\
\text { cambiar de ocupación para } \\
\text { mantener una proporción } \\
\text { de hombres y mujeres en } \\
\text { la ocupación igual a la que } \\
\text { se registra a nivel del total } \\
\text { de ocupados }\end{array}$ & $\begin{array}{l}\mathrm{D}=12 \mathrm{j}=1 \mathrm{JFjF}-\mathrm{MjM}{ }^{*} 100 \\
\text { Fj es el número de mujeres en la ocupación j, } \mathrm{F} \\
\text { es el total de mujeres ocupadas, Mj es el número } \\
\text { de hombres en la ocupación j y } \mathrm{M} \text { es el total de } \\
\text { hombres ocupados. }\end{array}$ & $\begin{array}{l}\text { Porcentaje del total de ocupados que debería cambiar de } \\
\text { ocupación para lograr una correspondencia exacta entre la } \\
\text { proporción de mujeres en cada ocupación y la proporción } \\
\text { de mujeres en el total de ocupados. } \\
\text { El índice de Duncan varía desde un valor mínimo de } 0 \text { (cero), } \\
\text { cuando no existe segregación y la proporción de mujeres es } \\
\text { igual en todas las ocupaciones, hasta un valor máximo de } 100 \\
\text { cuando existen sólo ocupaciones que son 100\% masculinas } \\
0100 \% \text { femeninas. }\end{array}$ \\
\hline $\begin{array}{l}\text { Índice de } \\
\text { contribución al } \\
\text { sexismo }\end{array}$ & $\begin{array}{l}\text { Expresa el grado en que } \\
\text { los grupos de un área del } \\
\text { conocimiento contribuye a } \\
\text { reproducir el sexismo en el } \\
\text { agregado. }\end{array}$ & $\begin{array}{l}\text { ICS }=(\text { Phd-Pmd)(Pha-Pma) } \\
\text { Phd es la participación de hombres en los grupos } \\
\text { de investigación en una disciplina, Pme es la } \\
\text { participación de mujeres en los grupos de inves- } \\
\text { tigación de una disciplina, Phe es la participación } \\
\text { de hombres en los grupos de investigación de toda } \\
\text { Colombia y Pme es la participación de mujeres } \\
\text { en los grupos de investigación en toda Colombia. }\end{array}$ & $\begin{array}{l}\text { Si el índice es positivo favorece la masculinización, si es } \\
\text { negativo favorece la feminización. Si es superior a la unidad } \\
\text { corresponde a un impacto más que proporcional con respecto } \\
\text { a la diferencia entre hombres y mujeres a nivel agregado. }\end{array}$ \\
\hline
\end{tabular}

2 Fuente: elaboración propia. 


\subsection{Indicadores}

Los indicadores calculados para el caso de los grupos de investigación en Economía y Administración, se presentan en el Tabla 1.

\section{Resultados}

\subsection{Panorama nacional}

En este apartado se presentan y analizan cifras sobre los grupos de investigación colombianos. La iniciativa de los grupos de investigación pretendía aunar esfuerzos para que las investigaciones se realizaran por un conjunto de investigadores con intereses en común, su número presenta un crecimiento significativo desde su conformación. En 2002 se registraron 544 y en 2010 había 4.707 grupos, y el 86,57\% de ellos se encontraban clasificados en la convocatoria de 2010 (Tabla 2).

Como se aprecia en la Ilustración 1 los grupos clasificados en categoría A1 han incrementado ligeramente su participación, la proporción de grupos categoría C ha permanecido estable entre 2008 y 2010 y los clasificados en categoría D se han incrementado al pasar de $36 \%$ a $41,45 \%$. Los grupos sin clasificar se han reducido desde 2008.

Tabla 2. Número de grupos de investigación por categoría $(2008-2010)^{3}$

\begin{tabular}{|l|c|c|c|}
\hline Grupos de investigación & $\mathbf{2 0 0 8}$ & $\mathbf{2 0 0 9}$ & $\mathbf{2 0 1 0}$ \\
\hline Categoría A1 & 135 & 203 & 216 \\
\hline Categoría A & 254 & 261 & 275 \\
\hline Categoría B & 671 & 647 & 668 \\
\hline Categoría C & 830 & 932 & 965 \\
\hline Categoría D & 1.481 & 1.905 & 1.951 \\
\hline Sub Total & 3.371 & 3.948 & 4.075 \\
\hline Sin Clasificar & 734 & 599 & $632^{*}$ \\
\hline Total & 4.105 & 4.547 & 4.707 \\
\hline
\end{tabular}

Ilustración 1. Número de grupos de investigación por categoría (2008-2010) ${ }^{4}$

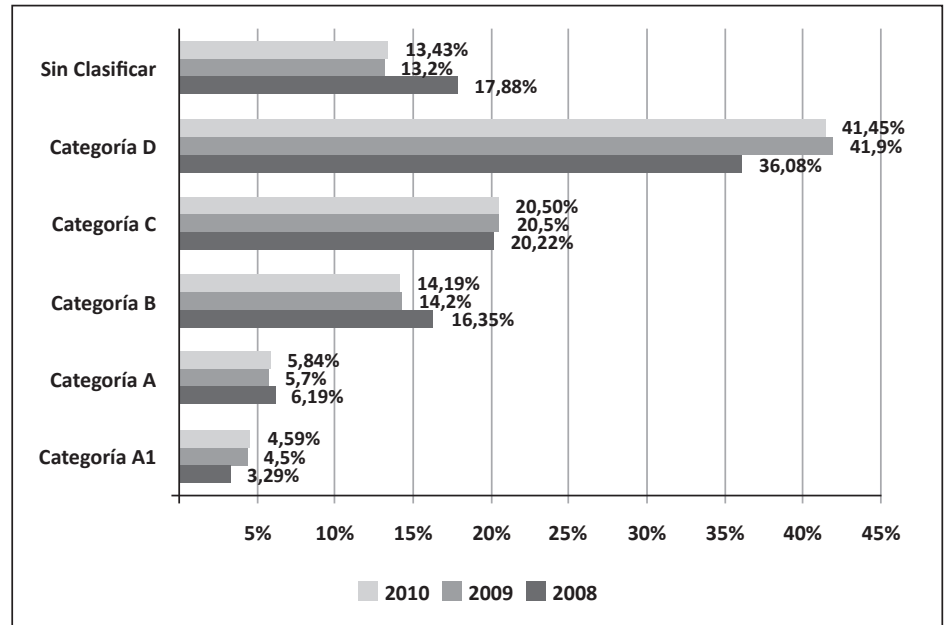

3 Fuente: Elaboración propia a partir de los datos de OCyT $(2007,2009)$ y Scienti.

Nota 1: 2010 es la convocatoria de medición más reciente.

Nota 2: * esta cifra corresponde a la información que se encontraba disponible en la plataforma Scienti en noviembre de 2011.

4 Fuente: elaboración propia a partir de los datos de OCyT (2009) y Scienti.

Nota 1: la cifra de grupos sin clasificar corresponde a la información que se encontraba disponible en la plataforma Scienti en noviembre de 2011. 
De otra parte, en el Tabla 3 puede analizarse la participación de la mujer como líder de los grupos de investigación. En todas las categorías son los hombres los que mayor participación tienen, $(66,4 \%)$ frente a $33,6 \%$ de las mujeres. Es importante mencionar que a medida que la clasificación del grupo mejora, se reduce la diferencia entre hombres y mujeres como líderes de grupos de investigación.

Si se realiza el análisis por disciplinas y no por la clasificación de los grupos de investigación, se encuentra que solamente en ciencias sociales, diseño de proyectos, economía doméstica, enfermería, fisioterapia, fonoaudiología, lingüística, microbiología, museología, nutrición, odontología, psicología, salud colectiva y servicio social, las mujeres son líderes de la mayoría de los grupos (ver Anexo 1).

En el caso de la distribución geográfica, se evidencia una mayor concentración de los grupos de investigación en Bogotá. En 2008, centralizaba el 44,32\% de los grupos (1.818 de 4.102) y en $201041,40 \%$ (1.949 de 4.707), como se ve en el tamaño de los semi-círculos grises y negros de la Ilustración 2.

Al hacer el análisis por los grupos de investigación según categorías, se encuentra que en todo el territorio nacional predominan los grupos categoría $\mathrm{D}$ y $\mathrm{C}$.
Los departamentos de la región oriental no cuentan con grupos de investigación.

Realizando un análisis de género, de acuerdo con el sexo de los líderes de grupo, se encuentra que la participación de los hombres es mayoritaria en todos los departamentos. No existe ningún territorio en el país, en el cual las mujeres superen en número a los hombres, en cuanto a la coordinación de grupos de investigación. No obstante, en las regiones de Bogotá, Antioquia y Valle del Cauca, la participación femenina es superior al del resto del país.

\subsection{Los grupos de investigación en Economía y Administración}

\subsubsection{Distribución vertical y horizontal}

En el área de economía, de total de grupos (202), el $23,3 \%^{9}$ (47) eran liderados por mujeres y el 76,7\% (155) por hombres. En el caso de administración el total de grupos correspondía a 243, el 29,21\% (71) tenían como líder a una mujer y el 70,79\% (172) a un hombre (Lis, 2012). En ambos casos el liderazgo femenino es inferior al promedio nacional (Tabla 4). En este caso se puede inferir que el liderazgo en los grupos de investigación en economía corresponde a un escenario masculino, dado que la participación de los hombres es superior al $60 \%$.

Tabla 3. Género del líder del grupo de investigación por categoría $(2010)^{5}$

\begin{tabular}{|l|c|c|c|c|c|}
\hline \multicolumn{1}{|c|}{ Grupo de Investigación } & Femenino & $\%$ & Masculino & $\%$ & Diferencia \\
\hline Categoría A1 & 57 & $1,2 \%$ & 159 & $3,4 \%$ & 102 \\
\hline Categoría A & 76 & $1,6 \%$ & 199 & $4,2 \%$ & 123 \\
\hline Categoría B & 206 & $4,4 \%$ & 462 & $9,8 \%$ & 256 \\
\hline Categoría C & 305 & $6,5 \%$ & 660 & $14,0 \%$ & 355 \\
\hline Categoría D & 752 & $16,0 \%$ & 1.199 & $25,5 \%$ & 447 \\
\hline Sin clasificación año 2010 & 184 & $3,9 \%$ & 448 & $9,5 \%$ & 263 \\
\hline Total general & 1.580 & $33,6 \%$ & 3.127 & $66,4 \%$ & 1.547 \\
\hline
\end{tabular}

5 Fuente: elaboración propia a partir de los datos de Scienti.

Nota 1: la cifra de grupos sin clasificar corresponde a la información que se encontraba disponible en la plataforma Scienti en noviembre de 2011.

9 Indicador de distribución horizontal. 
Ilustración 2. Grupos de investigación en Colombia 2008 vs. $2010^{6}$
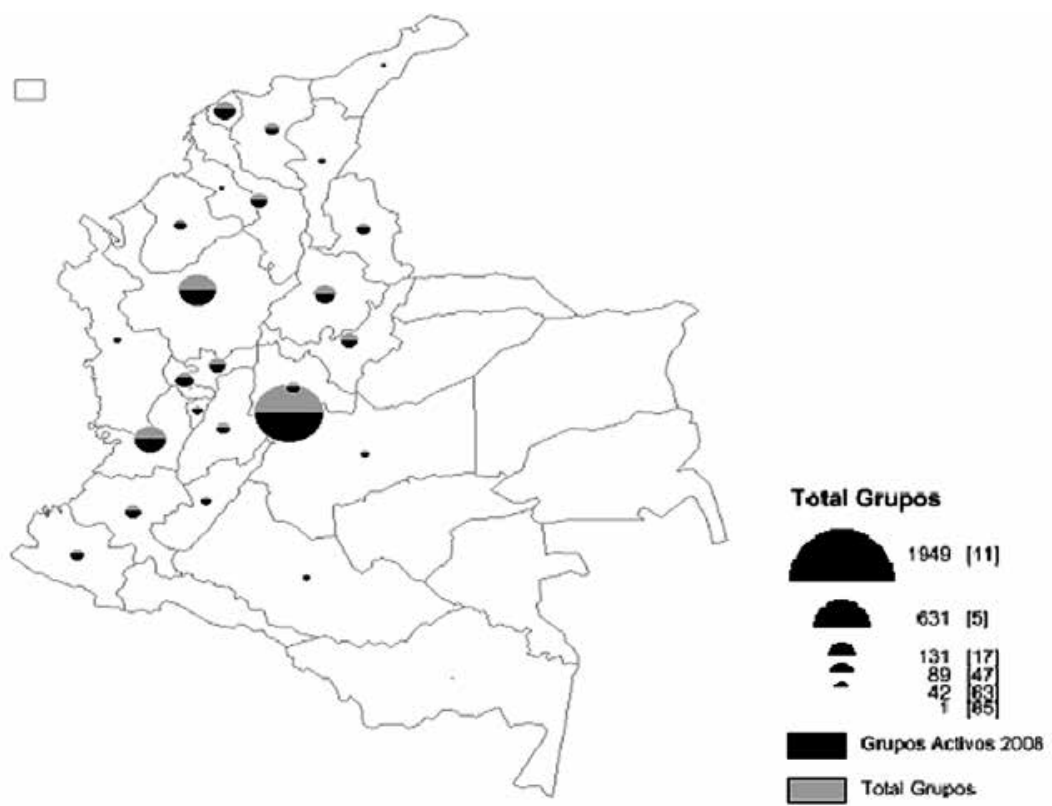

Ilustración 3. Sexo del líder del grupo de investigación por departamento (a noviembre de 2011)8
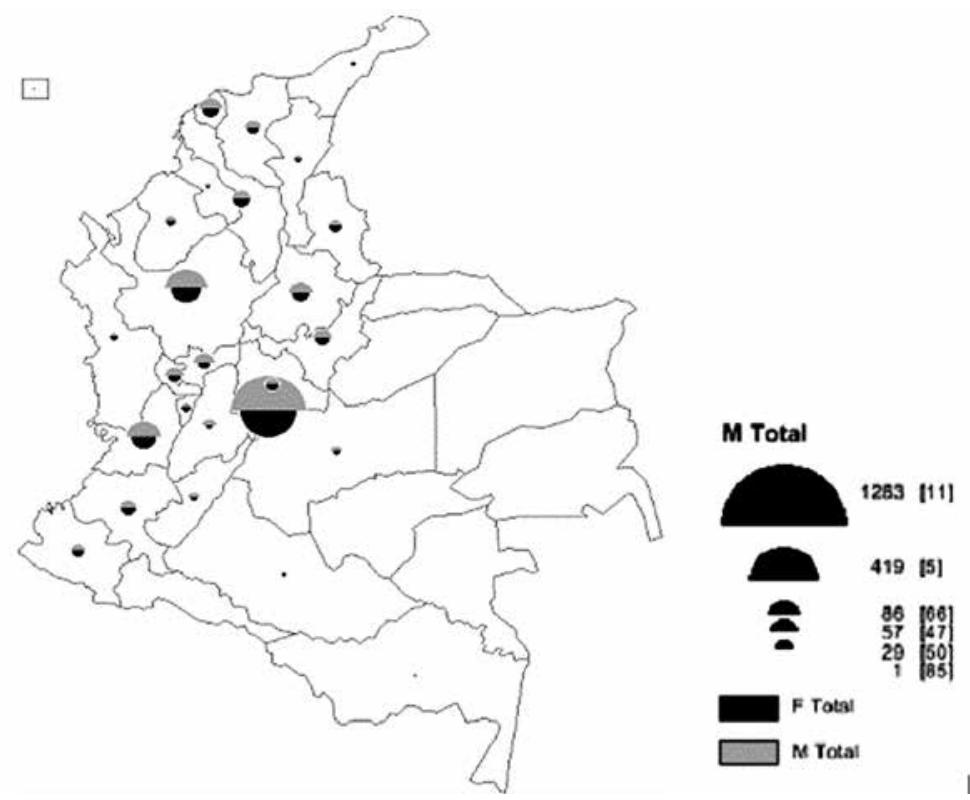

Fuente: elaboración propia usando Philcarto a partir de los datos de OcyT (2009), SIGOB y Scienti.

8 Fuente: elaboración propia usando Philcarto a partir de los datos de Scienti. 
Como se puede apreciar en la Tabla 4 en 22 de los 33 departamentos, en junio de 2012, había al menos un grupo de investigación en Economía. La mayor parte se concentraban en Bogotá (85), Antioquia (24), Valle del Cauca (17), Bolívar (15) y Santander (11). En el caso de Administración (Ilustración 4 y
Tabla 5), 21 departamentos contaban con la presencia de un grupo de investigación en esta área y al igual que en el caso de economía la mayor parte se encontraba en Bogotá (106), Valle del Cauca (37) y Antioquia (34).

Tabla 4. Sexo del líder de los grupos de investigación en Economía, por departamento ${ }^{11}$

\begin{tabular}{|c|c|c|c|c|}
\hline Departamento & Mujeres & Hombres & Total & $\begin{array}{c}\text { Diferencia entre } \\
\text { Hombres y } \\
\text { Mujeres }\end{array}$ \\
\hline Antioquia & 2 & 22 & 24 & 20 \\
\hline Atlántico & 2 & 5 & 7 & 3 \\
\hline Bolívar & 2 & 13 & 15 & 11 \\
\hline Boyacá & 1 & 6 & 7 & 5 \\
\hline Caldas & 1 & 1 & 2 & 0 \\
\hline Caquetá & 1 & 1 & 2 & 0 \\
\hline Cauca & 2 & 1 & 3 & -1 \\
\hline Cesar & - & 2 & 2 & 2 \\
\hline Chocó & - & 1 & 1 & 1 \\
\hline Córdoba & 1 & 2 & 3 & 1 \\
\hline Cundinamarca & - & 2 & 2 & 2 \\
\hline Distrito capital & 19 & 66 & 85 & 47 \\
\hline Huila & - & 1 & 1 & 1 \\
\hline Magdalena & 1 & 1 & 2 & 0 \\
\hline Meta & 4 & - & 4 & -4 \\
\hline Nariño & 1 & 1 & 2 & 0 \\
\hline Norte de Santander & - & 2 & 2 & 2 \\
\hline Quindío & 1 & 1 & 2 & 0 \\
\hline Risaralda & 1 & 5 & 6 & 4 \\
\hline Santander & 4 & 7 & 11 & 3 \\
\hline Sucre & 1 & - & 1 & -1 \\
\hline Tolima & - & 1 & 1 & 1 \\
\hline Valle & 3 & 14 & 17 & 11 \\
\hline
\end{tabular}

11 Fuente: elaboración propia a partir de los datos disponibles en Scienti hasta junio de 2012. 


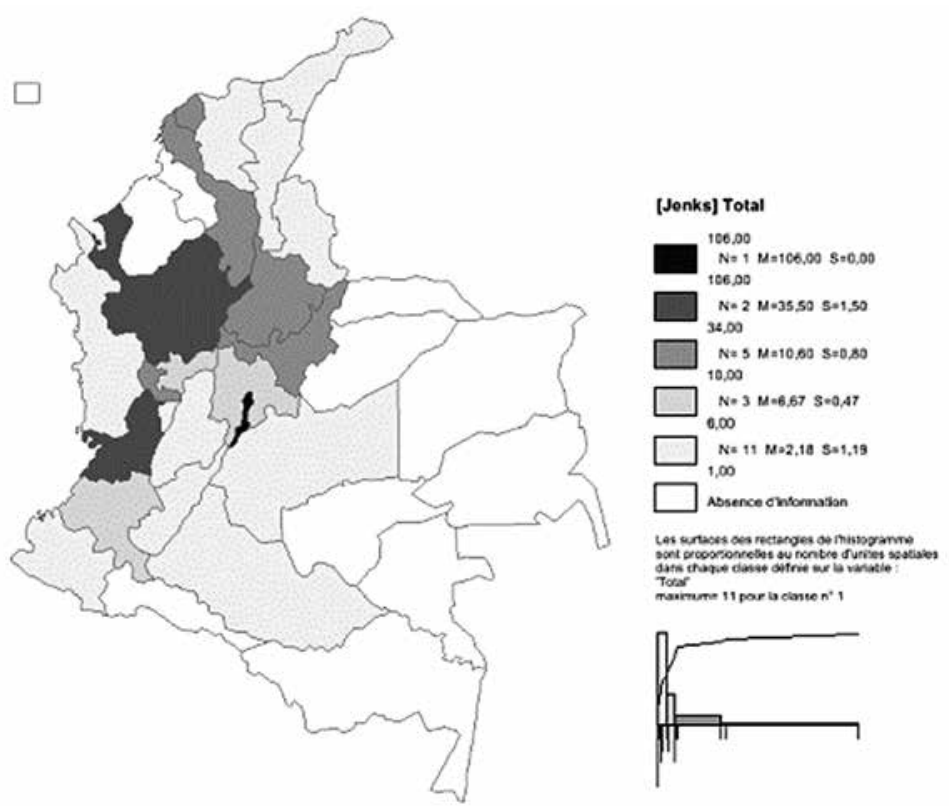

Ilustración 4. Distribución geográfica de los grupos de investigación en Administración ${ }^{12}$

Tabla 5. Sexo del líder de los grupos de investigación en Administración, por departamento (año 2011) ${ }^{13}$

\begin{tabular}{|c|c|c|c|c|}
\hline Departamento & Femenino & Masculino & Total & $\begin{array}{c}\text { Diferencia entre } \\
\text { Hombres y Mujeres }\end{array}$ \\
\hline Antioquia & 11 & 23 & 34 & 12 \\
\hline Atlántico & 4 & 8 & 12 & 4 \\
\hline Bolívar & 3 & 7 & 10 & 4 \\
\hline Boyacá & 4 & 6 & 10 & 2 \\
\hline Caldas & 2 & 5 & 7 & 3 \\
\hline Caquetá & - & 1 & 1 & 1 \\
\hline Cauca & 1 & 5 & 6 & 4 \\
\hline Cesar & - & 1 & 1 & 1 \\
\hline Choco & - & 1 & 1 & 1 \\
\hline Cundinamarca & 1 & 6 & 7 & 5 \\
\hline Distrito capital & 30 & 76 & 106 & 46 \\
\hline Huila & - & 2 & 2 & 2 \\
\hline La Guajira & 1 & - & 1 & -1 \\
\hline Magdalena & - & 4 & 4 & 4 \\
\hline Meta & 1 & - & 1 & -1 \\
\hline Nariño & 1 & 3 & 4 & 2 \\
\hline Norte de Santander & 2 & 1 & 3 & -1 \\
\hline Quindío & 1 & 2 & 3 & 1 \\
\hline Risaralda & 3 & 7 & 10 & 4 \\
\hline Santander & 4 & 7 & 11 & 3 \\
\hline Tolima & - & 3 & 3 & 3 \\
\hline Valle del Cauca & 15 & 22 & 37 & 7 \\
\hline
\end{tabular}

12 Fuente: elaboración propia usando Philcarto a partir de los datos disponibles en Scienti hasta junio de 2012.

13 Fuente: elaboración propia a partir de los datos disponibles en Scienti hasta junio de 2012. 
Si se desagrega la información del sexo del líder por departamento (Ilustración 5 y 6) se encuentran cin- co tipos de situaciones, las cuales se sintetizan en el Tabla 6.

Tabla 6. Tipología de departamentos, según sexo del líder de los grupos de investigación en economía y administración (junio de 2012) ${ }^{14}$

\begin{tabular}{|l|l|l|}
\hline \multicolumn{1}{|c|}{ Situaciones Relevantes } & Grupos de investigación en economía & \multicolumn{1}{|c|}{$\begin{array}{c}\text { Grupos de investigación } \\
\text { en administración }\end{array}$} \\
\hline No hay líderes de grupo mujeres & $\begin{array}{l}\text { Cesar, Chocó, Cundinamarca, Huila, Norte de San- } \\
\text { tander, Tolima }\end{array}$ & Caquetá, Cesar, Chocó, Huila, Magdalena y Tolima \\
\hline No hay líderes de grupo hombres & Sucre y Meta & La Guajira y Meta \\
\hline $\begin{array}{l}\text { Hay líderes de ambos sexos, pero superan en } \\
\text { cantidad las mujeres a los hombres }\end{array}$ & Cauca & Norte de Santander \\
\hline $\begin{array}{l}\text { Hay líderes de ambos sexos, pero superan en } \\
\text { cantidad los hombres a las mujeres }\end{array}$ & $\begin{array}{l}\text { Antioquia, Atlántico, Bolívar, Boyacá, Córdoba, Bo- } \\
\text { gotá, Risaralda, Santander y Valle del Cauca }\end{array}$ & $\begin{array}{l}\text { Antioquia, Atlántico, Bolívar, Boyacá, Caldas, Cau- } \\
\text { ca, Cundinamarca, Bogotá, Nariño, Quindío, Risa- } \\
\text { ralda, Santander y Valle del Cauca }\end{array}$ \\
\hline Hay paridad entre hombres y mujeres & Caldas, Caquetá, Magdalena, Nariño y Quindío & \\
\hline
\end{tabular}

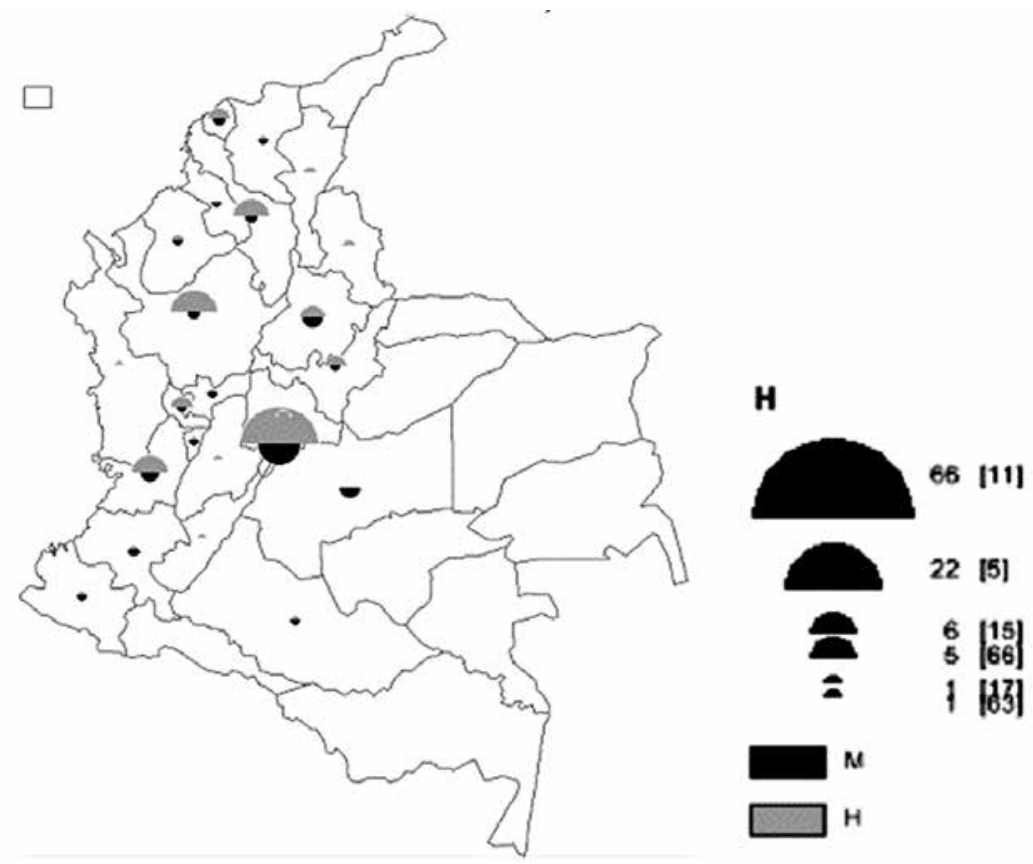

Ilustración 5. Sexo del líder de los grupos de investigación en Economía, por departamento (junio de 2012) ${ }^{15}$

14 Fuente: elaboración propia.

15 Fuente: elaboración propia usando Philcarto a partir de los datos disponibles en Scienti hasta junio de 2012. 
Ilustración 6. Sexo del líder de los grupos de investigación en Administración, por departamento (junio de 2012) ${ }^{16}$

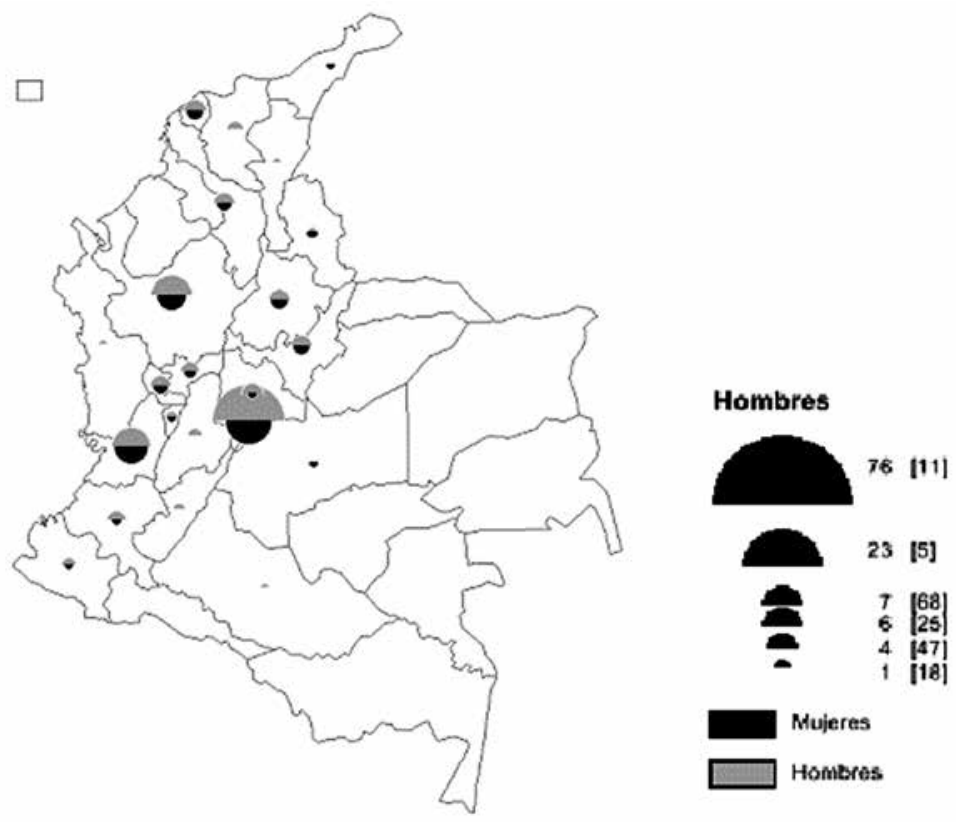

Ahora bien, si se analiza la distribución vertical de hombres y mujeres para los grupos del área de Economía, adicional al hecho de que la mayor parte de líderes de ambos sexos se concentran en grupos categoría D y sin clasificar, se aprecia que en el caso de las mujeres hay una distribución piramidal, en la cual ninguna es líder de un grupo de investigación clasificado en A1 (Tabla 7).
En los grupos del área de Administración, nuevamente la mayor parte de líderes de ambos sexos se concentran en grupos categoría $\mathrm{D}$, pero en este caso el segundo mayor porcentaje de mujeres se encuentra como líderes de grupos de la categoría $\mathrm{B}$, mientras que en el caso de los hombres esto se da en la categoría C (Tabla 8).

Tabla 7. Distribución vertical de la variable sexo del líder en grupos del área de Economía (junio de 2012) ${ }^{17}$

\begin{tabular}{|l|c|c|c|c|c|c|c|}
\cline { 2 - 8 } \multicolumn{1}{c|}{} & \multicolumn{7}{c|}{ Categoría grupos de investigación } \\
\cline { 2 - 8 } \multicolumn{1}{c|}{} & A1 & A & B & C & D & Sin clasificación año 2010 & Total \\
\hline Mujeres & - & 1 & 4 & 7 & 23 & 12 & 47 \\
\hline Distribución vertical & $0,00 \%$ & $2,13 \%$ & $8,51 \%$ & $14,89 \%$ & $48,94 \%$ & $25,53 \%$ & $100,00 \%$ \\
\hline Hombres & 8 & 4 & 23 & 28 & 54 & 38 & 155 \\
\hline Distribución vertical & $5,16 \%$ & $2,58 \%$ & $14,84 \%$ & $18,06 \%$ & $34,84 \%$ & $24,52 \%$ & $100,00 \%$ \\
\hline
\end{tabular}

16 Fuente: elaboración propia usando Philcarto a partir de los datos disponibles en Scienti hasta junio de 2012.

17 Fuente: elaboración propia a partir de los datos disponibles en Scienti hasta junio de 2012. 
Tabla 8. Distribución vertical de la variable sexo del líder en grupos del área de Administración (junio de 2012) ${ }^{18}$

\begin{tabular}{|l|c|c|c|c|c|c|c|}
\cline { 2 - 8 } \multicolumn{1}{c|}{} & \multicolumn{7}{c|}{ Categoría grupos de investigación } \\
\cline { 2 - 8 } \multicolumn{1}{c|}{} & A1 & A & B & C & D & Sin clasificación año 2010 & Total \\
\hline Mujeres & 2 & 2 & 12 & 10 & 40 & 5 & 71 \\
\hline Distribución vertical & $2,82 \%$ & $2,82 \%$ & $16,90 \%$ & $14,08 \%$ & $56,34 \%$ & $7,04 \%$ & 100 \\
\hline Hombres & 7 & 13 & 18 & 27 & 84 & 23 & 172 \\
\hline Distribución vertical & $4,07 \%$ & $7,56 \%$ & $10,47 \%$ & $15,70 \%$ & $48,84 \%$ & $13,37 \%$ & 100 \\
\hline
\end{tabular}

\subsection{2. Índice de feminidad y masculinidad}

Al considerar el índice de feminidad (IF) (Tabla 9), el valor calculado para los grupos del área de Economía es de 30,32 (es decir, por cada 100 hombres como líderes de grupos de investigación había 31 mujeres), para los grupos de Administración, esta cifra es más alta, el IF corresponde a 41,27 (es decir, por cada 100 hombres como líderes de grupos de investigación había 41 mujeres). En ambos casos el IF es inferior al agregado para todas las áreas (cercano a 52).

Al realizar este mismo análisis por categorías (Tabla 9), se encuentra que para los grupos del área de Economía el IF más alto se encuentra en la categoría D y que para las categorías $\mathrm{A}$ y $\mathrm{C}$ es igual. En el área de Administración, el IF más alto se encuentra en la categoría B y el más bajo en la clasificación A.
El índice de masculinidad (IM) agregado para el área de economía corresponde a 329 hombres por cada 100 mujeres que lideran los grupos de investigación y para el caso de los grupos de administración corresponde a 242. Esto quiere decir que en lo que respecta al liderazgo en grupos de investigación, el área de Economía es más masculinizada que Administración.

En Economía, el mayor IM se encuentra en el liderazgo de los grupos categoría B reportando un valor de 575 , lo que contrasta con el valor del índice para la misma categoría para Administración (150, el más bajo).

Los valores de los índices de feminidad y masculinidad contrastan con los indicadores de graduación universitaria. Entre 2001 y 2010, 30.752 personas obtuvieron su grado en Economía, de las cuales 17.939 eran mujeres, es decir, el 58,22\%. Esto implica un índice de feminidad de 140 (por cada 100

Tabla 9. Índice de feminidad y masculinidad para los grupos de investigación en Economía y Administración ${ }^{19}$

\begin{tabular}{|c|c|c|c|c|c|c|c|}
\hline \multirow{2}{*}{ Índice de } & \multicolumn{6}{|c|}{ Clasificación grupos de investigación } & \multirow{2}{*}{ Total } \\
\hline & A1 & A & B & C & D & Sin clasificación año 2010 & \\
\hline \multicolumn{8}{|c|}{ Economía } \\
\hline Feminidad & 0 & 25 & 17,39 & 25 & 42,59 & 31,58 & 30,32 \\
\hline Masculinidad & - & 400 & 575 & 400 & 234,78 & 316,67 & 329,79 \\
\hline \multicolumn{8}{|c|}{ Administración } \\
\hline Feminidad & 28,57 & 15,38 & 66,67 & 37,04 & 47,62 & 21,74 & 41,30 \\
\hline Masculinidad & 350 & 650 & 150 & 270 & 210 & 460 & 242,25 \\
\hline
\end{tabular}

18 Fuente: elaboración propia a partir de los datos disponibles en Scienti hasta junio de 2012.

19 Fuente: elaboración propia a partir de los datos disponibles en Scienti hasta junio de 2012. 
hombres graduados en economía se graduaron 140 mujeres entre 2001-2010). Por su parte, en el mismo período, 241.775 personas obtuvieron su grado en el área de administración, de las cuales 149.443 eran mujeres $(61,81 \%)$. Esto implica un índice de feminidad de 161,85 (por cada 100 hombres graduados en administración había 161 mujeres durante el período 2001-2010) (Lis \& Quiroz, 2012). Esto quiere decir, que a nivel de formación universitaria inicial (pregrado) se graduaron, en ambas disciplinas, más mujeres que hombres.

Para el caso de los posgrados, 130.571 personas obtuvieron un título de posgrado en el área de ciencias económicas (economía, administración y contaduría) entre 2000 y 2010. De estos, 69.413 fueron mujeres $(53.16 \%)$, lo que corresponde a un índice de feminidad de 113,5 (por cada 100 hombres posgraduados en ciencias económicas había 113 mujeres). En este caso, se aprecia una reducción en el IF, es decir, la participación de las mujeres en formación de posgrado en ciencias económicas se reduce.

Una posible explicación para que la relación de mujeres y hombres no se conserve en los grupos de investigación podría ser que si bien las mujeres se gradúan en mayor proporción que los hombres, no se dedican a actividades de investigación o docencia. Esta hipótesis se puede corroborar con la información sobre número de docentes universitarios por sexo. Para el segundo semestre de 2011, se aprecia que en Colombia, el número de docentes mujeres, para todos los tipos de IES es inferior al número de docentes hombres. En todos los casos la proporción de mujeres no es superior al $35 \%$.

\subsection{3. Índices de Duncan y de disimilaridad de Duncan}

Al aplicar el índice de Duncan para las categorías de los grupos de investigación, se encuentra que no hay segregación entre las clasificaciones, para las dos áreas del conocimiento, ya que el índice de Duncan es cercano a cero (Tabla 10). Para alcanzar una distribución equitativa $14,66 \%$ de los grupos en Economía, liderados por mujeres deberían mejorar su clasificación; en el caso de administración esta cifra es menor $(9,19 \%)$. Esto quiere decir que la distribución de mujeres como líderes de grupos de investigación en Administración es mejor, entre categorías de clasificación de los grupos, que en el caso del área de Economía.

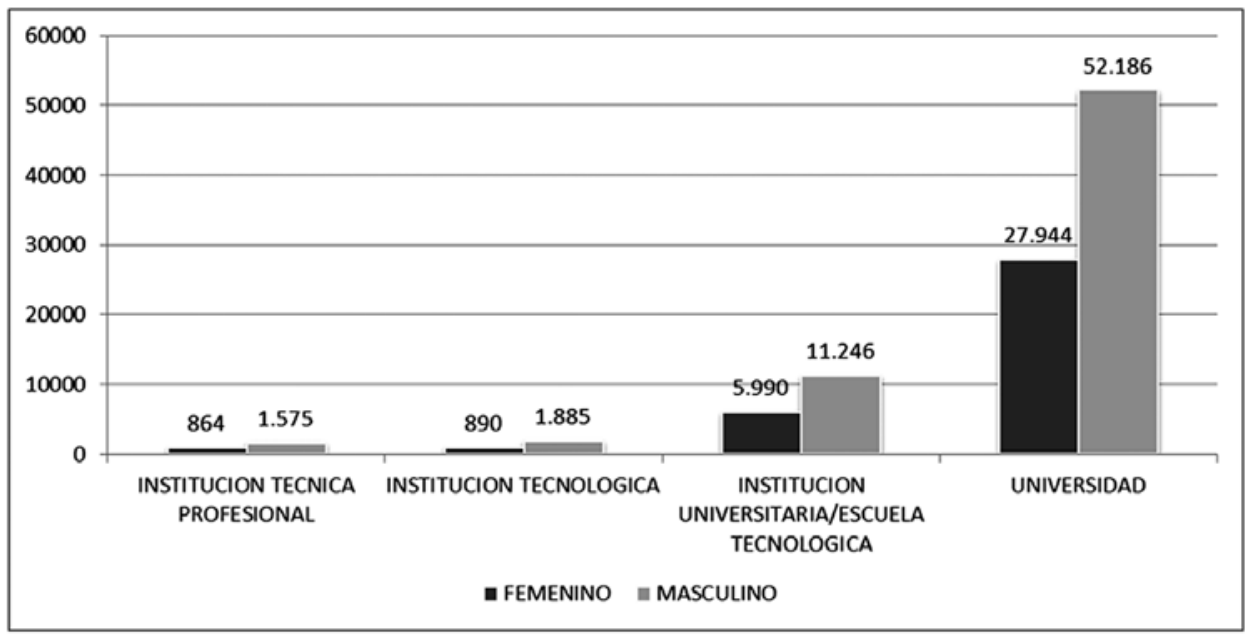

Ilustración 7. Número de docentes por sexo y tipo de institución (año 2011)20

20 Fuente: elaboración propia, con base en información del Ministerio de Educación Nacional. 
Tabla 10. Índice de Duncan ${ }^{21}$

\begin{tabular}{|l|c|c|c|c|c|c|c|}
\cline { 2 - 7 } & \multicolumn{7}{c|}{ Clasificación grupos de investigación } \\
\cline { 2 - 8 } & A1 & A & B & C & \multicolumn{2}{c|}{ D } & $\begin{array}{c}\text { Sin clasificación } \\
\text { año 2010 }\end{array}$ \\
\hline Distribución vertical femenina & 0,00 & 0,021 & 0,09 & 0,15 & 0,49 & 0,26 \\
\hline Distribución vertical masculina & 0,05 & 0,026 & 0,15 & 0,18 & 0,35 & 0,25 \\
\hline Diferencia & $-0,05$ & 0,005 & $-0,06$ & $-0,03$ & 0,14 & 0,01 \\
\hline & & Administración & & \\
\hline Distribución vertical & 0,03 & 0,03 & 0,17 & 0,14 & 0,56 & 0,07 \\
\hline Distribución vertical & 0,04 & 0,08 & 0,10 & 0,16 & 0,49 & 0,13 \\
\hline Diferencias & $-0,01$ & $-0,05$ & 0,06 & $-0,02$ & 0,08 & $-0,06$ \\
\hline
\end{tabular}

\subsection{4. Índice de contribución al sexismo (ICS)}

Este índice considera la diferencia en la participación de hombres y mujeres en el liderazgo de los grupos de investigación en cada área - Economía $(0,5346)$ y Administración $(0,4156)$-, con respecto a la diferencia en la participación de hombres y mujeres en el liderazgo de todos los grupos de investigación registrados en Colciencias $(0,318)$.

Para los grupos de economía, el resultado de este indicador es 1,68 y para administración 1.307. Al ser positivo implica que la distribución de hombres y mujeres líderes de los grupos, tiene un efecto sobre la masculinización de la actividad y al ser superior a la unidad se interpreta como un impacto más que proporcional con respecto a la diferencia en la participación entre hombres y mujeres a nivel agregado.

Ahora bien, como el ICS del área de administración es menor al de economía, implica que la distribución en el liderazgo de esta última tiene mayores efectos sobre la masculinización de la variable analizada en el agregado (total de grupos de todas las disciplinas) que la primera.
Hasta este momento se han descrito y comparado diferentes estadísticas e indicadores de género estudiando de forma transversal los grupos de investigación; no obstante, también es importante considerar de forma particular la estructura de cada grupo. Para ello, se propone como ejercicio -que puede ser ampliado a las otras categorías de clasificación de Colciencias- el análisis descriptivo y relacional para los grupos de investigación clasificados en categoría A1 y A en Economía.

\subsection{Los grupos de investigación clasificados en categoría A1 y A en Economía}

De acuerdo con la última clasificación de Colciencias (2010), en Economía sólo había 13 grupos de investigación $(6,44 \%$ de grupos del total la disciplina) categoría A1 (8) y A (5). Los grupos de estas categorías se ubican en 5 departamentos, la mayor parte en Bogotá y Antioquia (Tablas 11 y 12, e Ilustración 10). El grupo más antiguo surgió en 1984 (Grupo de Macroeconomía Aplicada de la Universidad de Antioquia) y los más recientes (cinco, tres de ellos ubicados en Bogotá) se conformaron desde el año 2000.

21 Fuente: elaboración propia a partir de los datos disponibles en Scienti hasta junio de 2012. 
Tabla 11. Ubicación de los grupos categoría A1 y A22

\begin{tabular}{|l|c|c|c|}
\hline \multicolumn{1}{|c|}{ Departamento } & A1 & A & $\begin{array}{c}\text { Total por } \\
\text { departamento }\end{array}$ \\
\hline Antioquia & 2 & 1 & 3 \\
\hline Atlántico & 1 & & 1 \\
\hline Bolívar & 1 & 1 & 2 \\
\hline Bogotá & 3 & 2 & 5 \\
\hline Valle & 1 & 1 & 2 \\
\hline Total por categoría & 8 & 5 & 13 \\
\hline
\end{tabular}

Al considerar las instituciones que avalan los grupos de investigación (Tabla 12), se aprecia que sólo la Universidad de Antioquia tiene dos grupos en estas categorías. Es relevante que ni la Universidad Nacional ni la Universidad de los Andes tengan algún grupo de investigación en esta clasificación.
Tabla 12. Instituciones que avalan los grupos de investigación clasificados en $\mathrm{A} 1$ y $\mathrm{A}$

\begin{tabular}{|l|l|c|c|}
\hline Departamento & \multicolumn{1}{|c|}{ Institución } & A1 & A \\
\hline \multirow{4}{*}{ Antioquia } & Universidad De Antioquia - Udea & 2 & - \\
\cline { 2 - 4 } & $\begin{array}{l}\text { Universidad Pontificia Bolivariana - Sede } \\
\text { Medellín }\end{array}$ & - & 1 \\
\hline \multirow{2}{*}{ Atlántico } & Universidad Del Norte - Uninorte & 1 & - \\
\hline \multirow{4}{*}{ Bolívar } & Universidad Tecnológica de Bolívar & - & 1 \\
\cline { 2 - 5 } & Universidad De Cartagena - Unicartagena & 1 & - \\
\hline \multirow{5}{*}{ Bogotá } & $\begin{array}{l}\text { Observatorio Colombiano De Ciencia Y } \\
\text { Tecnología - Ocyt }\end{array}$ & - & 1 \\
\cline { 2 - 5 } & Pontificia Universidad Javeriana & - & 1 \\
\cline { 2 - 5 } & Universidad De La Salle - Unisalle & 1 & - \\
\cline { 2 - 5 } & Universidad Del Rosario & 1 & - \\
\cline { 2 - 5 } & Universidad Externado de Colombia & 1 & - \\
\hline \multirow{2}{*}{$\begin{array}{l}\text { Valle del } \\
\text { Cauca }\end{array}$} & Universidad Autónoma De Occidente - Uao & 1 & - \\
\cline { 2 - 5 } & Universidad Del Valle - Univalle & - & 1 \\
\hline
\end{tabular}

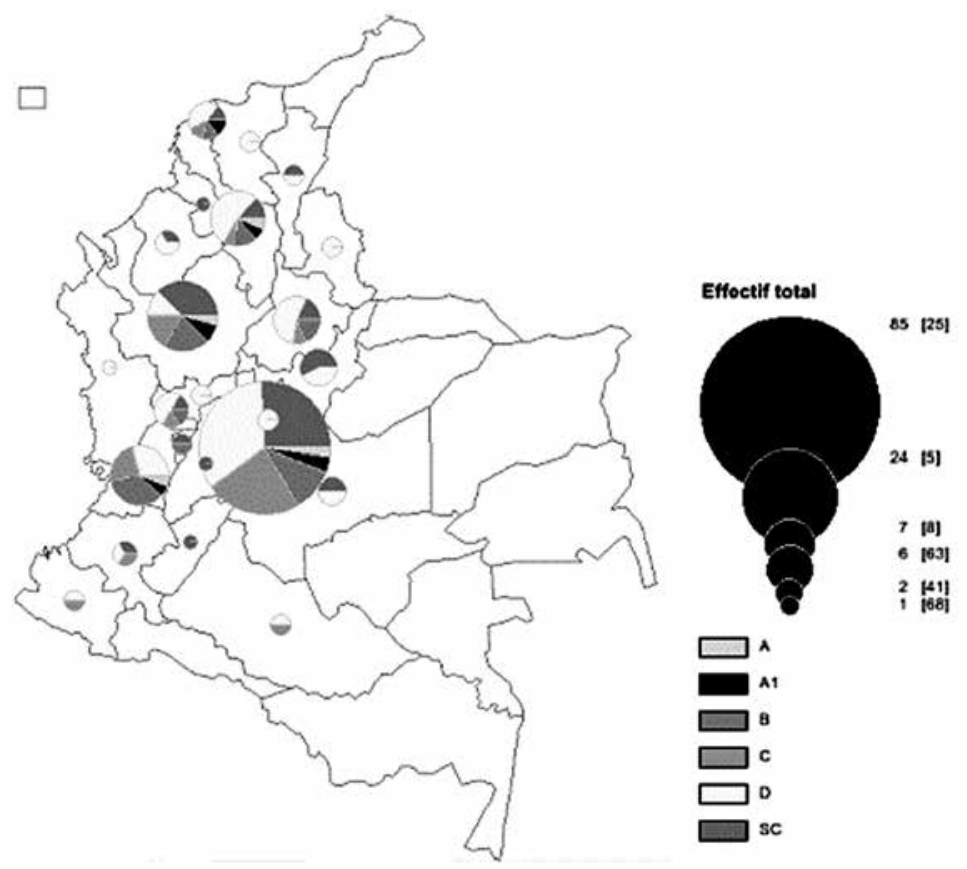

Ilustración 8. Ubicación de los grupos de investigación en Economía (junio de 2012)23

22 Fuente: elaboración propia a partir de los datos disponibles en Scienti hasta junio de 2012.

23 Fuente: elaboración propia usando Philcarto a partir de los datos disponibles en Scienti hasta junio de 2012. 
De acuerdo con la información del Tabla 7, ninguna mujer era líder de grupos de investigación A1 y sólo una (Mónica Salazar Acosta) lo era de un grupo categoría A (Sociedad, ciencia y tecnología en Colombia, del Observatorio Colombiano De Ciencia Y Tecnología).

Tabla 13 presenta la composición de los grupos por sexo y se aprecia que:

- $\quad$ Existe paridad entre los miembros activos (340) y no activos (335).

- La participación de las mujeres en el grupo de investigación correspondió al $31,5 \%$, lo que nuevamente permite identificar que se trata de un escenario masculinizado.

- Al considerar los investigadores activos (244) se evidencia que 69 son mujeres $(28,27 \%)$, que es superior a la participación en la participación de las mujeres en el liderazgo de los grupos en el área (23,3\%).

- Al analizar la distribución vertical se identifica que de las 107 mujeres vinculadas actualmente a los grupos de investigación $64,5 \%$ (69) son investigadoras, $33,6 \%$ (36) son estudiantes y $1,9 \%(2)$ son técnicas.

- La mayor parte de personas desvinculadas corresponde a estudiantes.
Ahora bien, a partir de la Ilustración 9 se evidencia que:

- En Bogotá no participan estudiantes hombres en los grupos de investigación.

- En ninguno de los departamentos los miembros femeninos vinculados superan a los miembros masculinos.

- Solamente en Bolívar y Antioquia hay técnicos vinculados.

- El mayor número de mujeres se encuentra en Bogotá (41 mujeres que equivalen al 33\% del total del personal de Bogotá), aunque en términos de participación es el Valle del Cauca donde hay mayor participación de mujeres $(34 \%, 16$ mujeres frente a 31 hombres).

El IF considerando todos los tipos de vinculación del personal activo corresponde a 45,92, es decir, por cada 100 hombres miembros activos de un grupo de investigación A1 y A en Economía hay 46 mujeres, esta cifra es superior al IF para la variable liderazgo de los grupos de investigación del área. El IM, por su parte, asciende a 217,75, el cual es inferior al calculado para el liderazgo de los grupos en la disciplina. El IF más alto se encuentra en la categoría estudiante y el IM más elevado en la categoría técnico (Tabla 14).

Tabla 13. Distribución de los miembros del grupo de investigación por tipo de vinculación y sexo (junio de 2012) ${ }^{24}$

\begin{tabular}{|c|c|c|c|c|c|c|}
\hline \multirow{2}{*}{ Activo } & Tipo de vinculación & Hombres & $\begin{array}{c}\text { Distribución } \\
\text { Horizontal Hombres }\end{array}$ & Mujeres & $\begin{array}{c}\text { Distribución } \\
\text { Horizontal Mujeres }\end{array}$ & $\begin{array}{c}\text { Total por tipo } \\
\text { de vinculación }\end{array}$ \\
\hline \multirow{4}{*}{ No } & Estudiante & 60 & $17,9 \%$ & 93 & $27,8 \%$ & 153 \\
\cline { 2 - 7 } & Investigador & 115 & $34,3 \%$ & 55 & $16,4 \%$ & 170 \\
\cline { 2 - 7 } & Técnico & 5 & $1,5 \%$ & 7 & $2,1 \%$ & 12 \\
\cline { 2 - 7 } & Total no activos & $\mathbf{1 8 0}$ & $53,7 \%$ & $\mathbf{1 5 5}$ & $46,3 \%$ & $\mathbf{3 3 5}$ \\
\hline \multirow{4}{*}{ Sí } & Estudiante & 52 & $15,3 \%$ & 36 & $10,6 \%$ & 88 \\
\cline { 2 - 7 } & Investigador & 175 & $51,5 \%$ & 69 & $20,3 \%$ & 244 \\
\cline { 2 - 7 } & Técnico & 6 & $1,8 \%$ & 2 & $0,6 \%$ & 8 \\
\cline { 2 - 7 } & Total activos & $\mathbf{2 3 3}$ & $68,5 \%$ & $\mathbf{1 0 7}$ & $31,5 \%$ & $\mathbf{3 4 0}$ \\
\hline
\end{tabular}

24 Fuente: elaboración propia a partir de los datos disponibles en Scienti hasta junio de 2012. 
Tabla 14. Índice de feminidad y masculinidad por tipo de vinculación y sexo25

\begin{tabular}{|l|c|c|c|c|}
\hline \multicolumn{1}{|c|}{ Vinculación } & Hombres & Mujeres & IF & IM \\
\hline Estudiante & 52 & 36 & 69,23 & 144,44 \\
\hline Investigador & 175 & 69 & 39,43 & 253,62 \\
\hline Técnico & 6 & 2 & 33,33 & 300 \\
\hline Total personal activo & 233 & 107 & 45,92 & 217,76 \\
\hline
\end{tabular}

Ilustración 9. Distribución de los miembros del grupo de investigación por tipo de vinculación, sexo y departamento²6

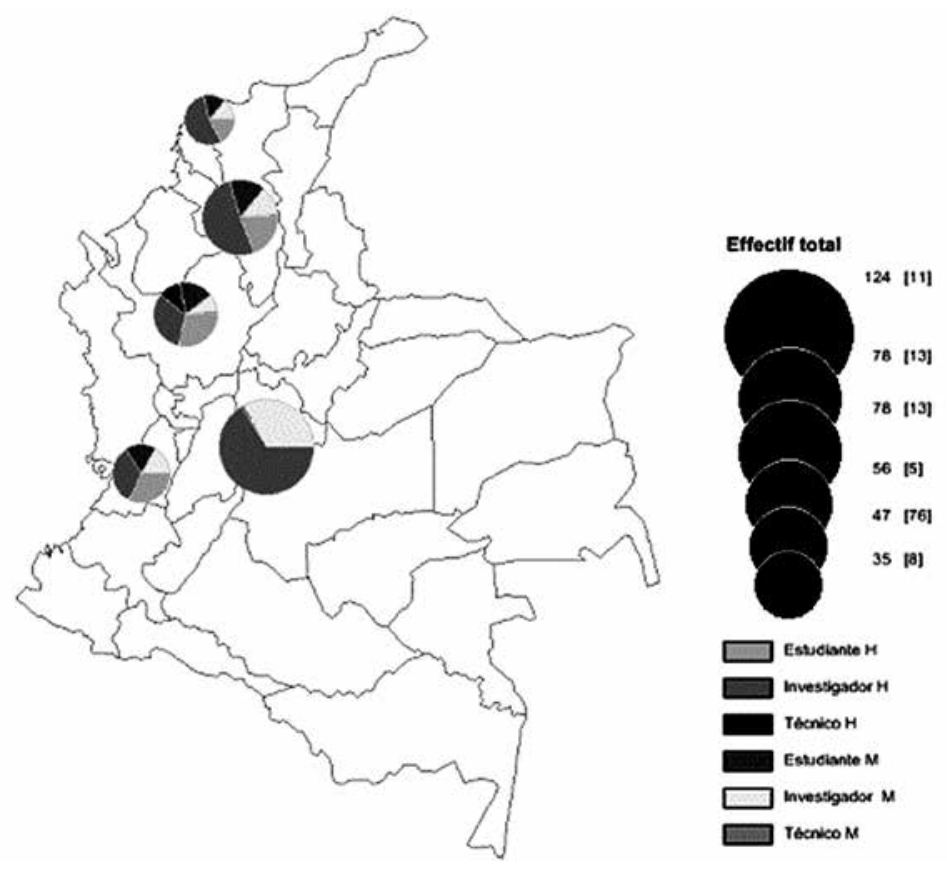

\section{Conclusiones}

A partir de las estadísticas e indicadores sobre grupos de investigación registrados en ScienTi fue posible comprobar tanto brechas de género como segregación geográfica en los grupos del área de Economía y Administración.

Se encontró que el liderazgo femenino en grupos de investigación en las dos áreas es inferior al promedio nacional. Lo anterior contrasta con las tasas de graduación de pregrado y posgrado entre 2001 y 2010, puesto que fueron las mujeres las que más se graduaron en pregrados de Economía, Administración y en posgrados de Ciencias Económicas, no obstante, no participan en actividades de investigación.

De otra parte, la mayor parte de grupos de investigación liderados por mujeres se encuentran en categoría $\mathrm{D}$, pero al calcular el indicador de Duncan, se

25 Fuente: elaboración propia a partir de los datos disponibles en Scienti hasta junio de 2012.

26 Fuente: elaboración propia a partir de los datos disponibles en Scienti hasta junio de 2012. 
establece que considerando los resultados de clasificación y el liderazgo por sexo no hay una alta segregación en la clasificación.

Con respecto a la segregación espacial, se estableció que el $66,66 \%$ de los departamentos colombianos hay al menos un grupo de investigación en Economía, y en $64,7 \%$ de los entes territorial al menos uno en Administración. No obstante, la mayor parte se concentra en Bogotá, Antioquia y Valle del Cauca, lo cual coincide con los resultados agregados para Colombia. Así mismo, en la mayoría de los departamentos hay líderes de grupo de ambos sexos, pero superan en cantidad los hombres a las mujeres.

Al analizar la composición de los grupos de investigación clasificados en categoría A1 y A del área de Economía, las brechas de género y la segregación geográfica se mantienen. No obstante, los indicadores de paridad de género son mejores, al compararlos con los resultados obtenidos al analizar el liderazgo de los grupos de investigación de la disciplina.

Para seguir profundizando en este análisis es importante, emplear variables adicionales. Podrían considerarse para futuros trabajos: análisis de la composición de cada grupo de investigación por sexo, formación, horas de investigación declaradas, tipo de productos, destino de las publicaciones, redes de colaboración, entre otros.

\section{Referencias}

Arango, L. (2006). Jóvenes en la universidad: género clase e identidad profesional. Bogotá: Siglo XXI Editores.

Bailyn, L. (2003). Academic Careers and Gender Equity: Lessons Learned from MIT. En: Gender, Work and Organisation, 10(2), 137-153.

Buquet Corleto, A., Cooper, J., Rodríguez, H. \& Botello, L. (2006). Presencia de mujeres y hombres en la UNAM: una radiografía. México: UNAM.

Buquet Corleto, A., Cooper, J. \& Rodríguez, H. (2010). Sistema de indicadores para la equidad de género en instituciones de educación superior. México: UNAM. Disponible en: http://www. pueg.unam.mx/images/stories/Equidad/Investigacion/sist.\%20 de\%20indicadores.pdf

Buquet Corleto, A., Hernández, A. \& Jiménez, V. (2011). Diagnóstico de la situación de mujeres y hombres por dependencia. Instituto de Matemáticas de la UNAM. México D.F.: UNAM.

Bustos, O. \& Blázquez, N. (2003). Qué dicen las académicas acerca de la UNAM. México: UNAM.
Cano, C. \& Orozco, M. (2011). Diferenciales Salariales en el Área Metropolitana de Medellín, a través de Regresiones por Cuantiles en el período 2002-2006. En: Ecos de Economia, 15 (32): 59-97.

Cepeda, L. \& Barón, J. (2012). Segregación educativa y la brecha salarial por género entre los recién graduados universitarios en Colombia (Documentos de trabajo sobre economía regional, 162). Cartagena: Banco de la República de Colombia.

Daza-Caicedo, S. (2010). Las mujeres en el SNCTI. Balance de una década en condiciones diferentes. OCyT, Indicadores de Ciencia y Tecnología (pp. 279-316). Bogotá: OCyT.

Daza-Caicedo, S \& Pérez-Bustos, T. (2008). Contando mujeres. Una reflexión sobre los indicadores de género y ciencia en Colombia. En: Revista de Antropología y sociología Virajes, 10: 29-51.

Escolano, E. (2006). Discriminación en un medio meritocrático: las profesoras en la Universidad española. En: Revista Mexicana de Sociología, 2: 231-263.

Escolano, E. (2009). El poder como asignatura pendiente de las académicas en las universidades españolas. En: Chávez Gutiérrez, M., et al. (eds). Género y trabajo en las universidades (pp. 83-127). Guadalajara: Universidad de Guadalajara.

García Guevara, P. (1992). Notas sobre la participación de la mujer académica en la Universidad de Guadalajara. En: Revista Tiempo de Ciencia, 28: 33-36.

García Guevara, P. (2004). Mujeres académicas: El caso de una universidad estatal mexicana. México: Plaza y Valdés. Universidad de Guadalajara.

García Guevara, P. (2009). Las académicas entre la materialidad política y la subjetividad. En: Chávez Gutiérrez, M., et al. (eds). Género y trabajo en las universidades (pp. 31-48). Guadalajara: Universidad de Guadalajara.

González-García, M. \& Pérez Sedeño, E. (2002). Ciencia, tecnología y género. En: Revista Iberoamericana de ciencia, tecnología, sociedad e innovación. 2 (2).

Gutiérrez, A. (2010). Educación y mercado laboral en Santander: una mirada en perspectiva de género. En: Revista Lebret, 2(2): 47-69.

Lemarchand, G. (ed.) (2010). Sistemas Nacionales de ciencia, tecnología e innovación en América Latina y el Caribe. Montevideo: Unesco.

Lis Gutiérrez, J. (2012). Algunos indicadores de género aplicados a los grupos de investigación colombianos en administración. En: I Encuentro Regional de Investigación en Estudios Sociales desde las Ciencias Económicas, Administrativas y Contables, 26 y 27 de julio de 2012. Bogotá: Universidad Militar.

Lis Gutiérrez, J. \& Quiroz Rubiano, M. (2012). Perspectiva de género aplicada a la graduación y posgraduación universitaria en Administración y Economía (2001-2010). Documento Presentado en la VIII Semana Económica. Universidad Central, 11 al 13 de septiembre. Bogotá, Colombia.

Monge, R. V. (2006). Guía de indicadores para la evaluación del impacto de género de las políticas públicas. Açores, Madeira: FEDER. Disponible en: http://www.funiovi.org/c/ document_library/get_file?uuid=f169fbf4-9028-4f2a-b834c43bd832056a\&group $\overline{I d}=41671$.

Munévar, D., Arana, I. \& Agudelo, C. (2006). Productividad Académica en la Universidad Nacional. Una aproximación crítica. Bogotá: Universidad Nacional de Colombia.

OCyT (2007). Evaluación de las actividades de comunicación pública de la Ciencia y la tecnología en el sistema nacional de ciencia y Tecnología colombiano. 1990-2004. Bogotá: Colciencias. 
Disponible en: http://www.ocyt.org.co/html/archivosProyectos/ Informe final.pdf. Consultado el 15 de marzo de 2011.

OCyT (2009). Indicadores de Ciencia y Tecnología. Edición de Bolsillo. Bogotá. Colciencias.

Olaya, D. (2003). La creciente participación de la mujer en la investigación en ciencia y tecnología a finales de la última década. En: Colombia, Ciencia y Tecnología, 21(1): 34-46.

Palomar, C. (2004). La incorporación de la perspectiva de género en la Universidad de Guadalajara. En: GénEros, 34 (11): 16-21.

Papadópulos, J. \& R. Radakovich. (2006). Educación superior y género en América Latina y el Caribe. En: Informe sobre la Educación Superior en América Latina y el Caribe 2000-2005. La metamorfosis de la educación superior. Caracas: UNESCO, IESALC.
Tovar, P. (2002). Género y ciencia en Colombia: Algunos indicadores. Colombia Ciencia y Tecnología, 20 (2): 3-10.

Tovar, P. (2004). Indicadores nacionales de género, ciencia y tecnología. En: Memorias seminario: Las mujeres colombianas en el sistema de ciencia y tecnología: obstáculos y logros. Bogotá: ICANH.

Tovar, P. (2005). La percepción que tienen los colombianos sobre la ciencia y la tecnología: la importancia de tener una perspectiva de género. En: Aguirre, J. (ed.). La percepción que tienen los colombianos sobre la ciencia y la tecnología. Bogotá: Colciencias.

UNESCO (2009). S\&T World Data Fact Sheet. Montreal: Unesco.

Zapata, M. (2010). La equidad de género en las universidades alemanas. En: Mingo, A. Desasosiegos. Relaciones de género en la educación (pp. 109-150). México D.F.: Instituto de Investigaciones sobre la Universidad y la Educación, UNAM. 


\section{ANEXO 1}

Sexo del líder del grupo de investigación por área $(2011)^{27}$

\begin{tabular}{|c|c|c|c|c|}
\hline Área & Mujeres & Hombres & Total & $\begin{array}{c}\text { Diferencia } \\
\text { (H-M) }\end{array}$ \\
\hline Administración & 72 & 172 & 244 & 100 \\
\hline Administración hospitalaria & 1 & 2 & 3 & 1 \\
\hline Agronomía & 24 & 70 & 94 & 46 \\
\hline Antropología & 17 & 27 & 44 & 10 \\
\hline Arqueología & 2 & 6 & 8 & 4 \\
\hline Arquitectura y Urbanismo & 28 & 36 & 64 & 8 \\
\hline Artes & 18 & 25 & 43 & 7 \\
\hline Astronomía & & 6 & 6 & 6 \\
\hline Biofísica & 1 & 2 & 3 & 1 \\
\hline Biología General & 46 & 60 & 106 & 14 \\
\hline Biomedicina & & 1 & 1 & 1 \\
\hline Bioquímica & 8 & 10 & 18 & 2 \\
\hline Botánica & 5 & 15 & 20 & 10 \\
\hline Ciencia de la Computación & 32 & 112 & 144 & 80 \\
\hline Ciencia de la Información & 6 & 12 & 18 & 6 \\
\hline Ciencia Política & 19 & 49 & 68 & 30 \\
\hline Ciencia y Tecnología de Alimentos & 18 & 29 & 47 & 11 \\
\hline Ciencias & 3 & 8 & 11 & 5 \\
\hline Ciencias Estadísticas & & 2 & 2 & 2 \\
\hline Ciencias Sociales & 9 & 8 & 17 & -1 \\
\hline Comunicación & 26 & 33 & 59 & 7 \\
\hline Demografía & 2 & 4 & 6 & 2 \\
\hline Derecho & 70 & 171 & 241 & 101 \\
\hline Diseño de Proyectos & 4 & & 4 & -4 \\
\hline Diseño Industrial & 9 & 14 & 23 & 5 \\
\hline Ecología & 20 & 55 & 75 & 35 \\
\hline Economía & 47 & 155 & 202 & 108 \\
\hline Educación & 201 & 208 & 409 & 7 \\
\hline Educación Física & 4 & 11 & 15 & 7 \\
\hline Enfermería & 22 & 1 & 23 & -21 \\
\hline Estudios Sociales & 2 & 2 & 4 & 0 \\
\hline
\end{tabular}

27 Fuente: elaboración propia a partir de los datos de Scienti.

Nota 1: la cifra de grupos sin clasificar corresponde a la información que se encontraba disponible en la plataforma Scienti en noviembre de 2011. 


\begin{tabular}{|c|c|c|c|c|}
\hline Área & Mujeres & Hombres & Total & $\begin{array}{c}\text { Diferencia } \\
(\mathrm{H}-\mathrm{M})\end{array}$ \\
\hline Farmacia & 10 & 10 & 20 & 0 \\
\hline Farmacología & & 5 & 5 & 5 \\
\hline Filosofía & 31 & 89 & 120 & 58 \\
\hline Física & 16 & 97 & 113 & 81 \\
\hline Fisiología & 1 & 6 & 7 & 5 \\
\hline Fisioterapia y Terapia Ocupacional & 23 & 5 & 28 & -18 \\
\hline Fonoaudiología & 4 & 1 & 5 & -3 \\
\hline Genética & 15 & 22 & 37 & 7 \\
\hline Geociencias & 11 & 50 & 61 & 39 \\
\hline Geografía & 1 & 7 & 8 & 6 \\
\hline Historia & 20 & 42 & 62 & 22 \\
\hline Ingeniería Aeroespacial & 1 & 5 & 6 & 4 \\
\hline Ingeniería Agrícola & 2 & 8 & 10 & 6 \\
\hline Ingeniería Biomédica & 3 & 22 & 25 & 19 \\
\hline Ingeniería Civil & 23 & 67 & 90 & 44 \\
\hline Ingeniería de materiales y metalúrgica & 5 & 29 & 34 & 24 \\
\hline Ingeniería de Minas & 2 & 10 & 12 & 8 \\
\hline Ingeniería de Producción & 29 & 88 & 117 & 59 \\
\hline Ingeniería de transporte & 2 & 8 & 10 & 6 \\
\hline Ingeniería Eléctrica & 15 & 143 & 158 & 128 \\
\hline Ingeniería Mecánica & 5 & 60 & 65 & 55 \\
\hline Ingeniería Mecatrónica & & 4 & 4 & 4 \\
\hline Ingeniería Naval y océanica & & 2 & 2 & 2 \\
\hline Ingeniería Química & 14 & 35 & 49 & 21 \\
\hline Ingeniería Sanitaria & 21 & 23 & 44 & 2 \\
\hline Inmunología & & 7 & 7 & 7 \\
\hline Letras & 6 & 14 & 20 & 8 \\
\hline Lingüística & 33 & 20 & 53 & -13 \\
\hline Medicina & 115 & 220 & 335 & 105 \\
\hline Medicina Veterinaria & 7 & 34 & 41 & 27 \\
\hline Microbiología & 28 & 24 & 52 & -4 \\
\hline Morfología & 1 & 1 & 2 & 0 \\
\hline Multidisciplinar & 32 & 69 & 101 & 37 \\
\hline Museología & 1 & & 1 & -1 \\
\hline Nutrición & 6 & & 6 & -6 \\
\hline Oceanografía & 2 & 6 & 8 & 4 \\
\hline Odontología & 25 & 17 & 42 & -8 \\
\hline Parasitología & 1 & 7 & 8 & 6 \\
\hline
\end{tabular}




\begin{tabular}{|l|c|c|c|c|}
\hline \multicolumn{1}{|c|}{ Área } & Mujeres & Hombres & Total & $\begin{array}{c}\text { Diferencia } \\
\text { (H-M) }\end{array}$ \\
\hline Planeamiento Urbano y regional & 14 & 32 & 46 & 18 \\
\hline Probabilidad y Estadística & 26 & 77 & 103 & 51 \\
\hline Psicología & 104 & 70 & 174 & -34 \\
\hline Química & 39 & 73 & 112 & 34 \\
\hline Química Industrial & & 1 & 1 & 1 \\
\hline Recursos Forestales y & 3 & 11 & 14 & 8 \\
\hline $\begin{array}{l}\text { Recursos Pesqueros e } \\
\text { Ingeniería de Pesca }\end{array}$ & 2 & 11 & 13 & 9 \\
\hline Relaciones Internacionales & 1 & 4 & 5 & 3 \\
\hline Salud Colectiva & 59 & 50 & 109 & -9 \\
\hline Servicio Social & 34 & 12 & 46 & -22 \\
\hline Sociología & 16 & 36 & 52 & 20 \\
\hline Teología & 3 & 18 & 21 & 15 \\
\hline Turismo & 4 & 4 & 8 & 0 \\
\hline Zoología & 10 & 14 & 24 & 4 \\
\hline Zootecnia & 11 & 24 & 35 & 13 \\
\hline
\end{tabular}

